\title{
PERDA ENDÓGENA E ABSORÇÃO REAL DE FÓSFORO EM DIETAS PARA EQÜINOS EM CRESCIMENTO'
}

\author{
CARLOS EDUARDO FURTADO ${ }^{2}$, HUGO TOSI ${ }^{3}$ e DORINHA MIRIAM SILBER SCHMIDT VITTI ${ }^{4}$
}

\begin{abstract}
RESUMO - O trabalho teve por objetivo avaliar os efeitos de diferentes níveis de $\mathrm{P}$ na dieta de eqüinos em crescimento sobre sua perda endógena fecal, e verificar qual seja a exigência mínima diária desse elemento na sua alimentação. Foram utilizados 16 eqüinos machos em crescimento, recebendo dieta basal sem suplementação de $\mathrm{P}$, e dieta basal suplementada com fosfato bicálcico, para fornecer $15,20 \mathrm{e}$ $25 \mathrm{~g} \mathrm{P} /$ animal/dia. No $16^{\mathrm{o}}$ dia experimental, foram injetados $30 \mathrm{MBq}$ de ${ }^{32} \mathrm{P} /$ animal e coletaram-se amostras de sangue, fezes e urina, durante sete dias. Foram determinadas as atividades específicas do $\mathrm{P}$ no plasma, nas fezes e na urina, e calculou-se a perda endógena fecal e a absorção real de P. A perda endógena fecal e a absorção real de $\mathrm{P}$ não foram afetadas $(\mathrm{P}>0,05)$ pelos tratamentos, e foram estimados, em média, 10,34 mg P/kg PV/dia e 47,07\%, respectivamente, o que indica que, nas condições experimentais, animais com idade média de 19 meses necessitam de 21,96 mg de P/kg PV/dia para manter o balanço da perda metabólica fecal, e a quantidade diária de P de 14,75 g.
\end{abstract}

Termos para indexação: cavalos, dieta, necessidades nutricionais, nutrientes minerais, perdas nutricionais, defecação, nutrição animal.

\section{ENDOGENOUS LOSS AND TRUE ABSORPTION OF PHOSPHORUS IN THE DIET FOR GROWING HORSES}

\begin{abstract}
The objective of this work was to evaluate the effects of different P levels in the alimentary diet of growing horses on the endogenous fecal losses, and to verify the real daily minimum requirement of this element for their feeding. Sixteen male horses receiving a basal diet with no phosphorus supplementation and basal diet supplemented with dicalcium phosphate to provide $\mathrm{P}$ intake of 15,20 and $25 \mathrm{~g} /$ animal/day were used. At the $16^{\text {th }}$ day of the trial, each horse was intravenously injected with $30 \mathrm{MBq}$ of ${ }^{32} \mathrm{P} / \mathrm{animal}$, and blood, feces and urine were collected for seven days at 24 hours intervals. The specific activities of the radiophosphorus in the plasma, feces and urine were determined and the fecal endogenous loss and true phosphorus absorption were calculated. Fecal endogenous loss and true phosphorus absorption were unaffected $(\mathrm{P}>0.05)$ by $\mathrm{P}$ levels, and the average of $10.34 \mathrm{mg} \mathrm{P} / \mathrm{kg} / \mathrm{BW} /$ day and $47.07 \%$, respectively, was estimated. Therefore, the horses in this experiment required $21.96 \mathrm{mg} \mathrm{P} / \mathrm{kg} / \mathrm{BW} /$ day to balance fecal endogenous loss, and phosphorus daily requirement of $14.75 \mathrm{~g}$.

Index terms: horses, diet, nutritional requirements, mineral nutrients, nutritional losses, defecation, animal nutrition.
\end{abstract}

\footnotetext{
${ }^{1}$ Aceito para publicação em 22 de novembro de 1999.

Extraído da tese de doutorado do primeiro autor. Projeto financiado pela FAPESP.

${ }^{2}$ Zoot., D.Sc., Prof. Associado, Centro de Ciências Agrárias, Dep. de Zootecnia, Universidade Estadual de Maringá (UEM), CEP 87020-900 Maringá, PR. E-mail: cefurtado@uem.br

${ }^{3}$ Eng. Agrôn., D.Sc., Prof. Titular, Dep. de Zootecnia de Não-Ruminantes, Fundação Carlos Alberto Vanzolini (FCAV), Universidade Estadual Paulista (UNESP), Caixa Postal 390, CEP 13470-000 Jaboticabal, SP. E-mail: tosi@fcav.unesp.br

${ }^{4}$ Biól., D.Sc., Centro de Energia Nuclear na Agricultura (CENA), Universidade de São Paulo (USP), Caixa Postal 96, CEP 13400-000 Piracicaba, SP. E-mail: vitti@cena.usp.br
}

\section{INTRODUÇ̃̃O}

O P é um elemento mineral de grande importância para a manutenção das funções vitais, não apenas compondo estruturas, mas também participando de reações bioquímicas no organismo animal (National Research Council, 1989).

Os principais estudos de metabolismo de $\mathrm{P}$ em eqüinos foram realizados nos Estados Unidos, no início da década de 70 , utilizando pôneis, nos quais foi avaliada a perda endógena fecal desse elemento, sem a qual não se pode determinar com precisão as 
exigências do animal (Schryver et al., 1971; Hintz et al., 1973; Cymbaluk, 1990).

A determinação da exigência animal desse elemento reveste-se de fundamental importância para fazer com que a suplementação de P seja a mais eficiente possível. As pesquisas em eqüinos a este respeito são escassas, em comparação com o universo de publicações correlatas em outras espécies (Cuddeford, 1991).

Segundo Ott (1995), as exigências nutricionais dos cavalos são compostas por duas frações: necessidades de manutenção e necessidades de atividade (gestação, lactação, crescimento e trabalho). Estas frações são aditivas, e ambas devem ser fornecidas para a manutenção e composição do peso vivo.

$\mathrm{O}$ número relativamente reduzido de pesquisas no Brasil, em nutrição de eqüinos, conduz à consulta de tabelas estrangeiras, que, na maioria das vezes, não são aplicáveis às nossas condições.

O estudo teve como objetivo avaliar os efeitos de diferentes níveis de $\mathrm{P}$ na dieta alimentar dos eqüinos em crescimento sobre sua perda endógena fecal, e verificar a exigência mínima desse elemento na sua alimentação.

\section{MATERIAL E MÉTODOS}

O experimento foi realizado no setor de Eqüinocultura da FCAV-UNESP, câmpus de Jaboticabal, SP, e no setor de Ciências Animais do CENA-USP, câmpus de Piracicaba, SP. Utilizaram-se 16 eqüinos machos da raça Brasileiro de Hipismo com idade média de 19 meses e $322,56 \pm 23,9 \mathrm{~kg}$. Foram estudados quatro níveis (tratamentos) de P: dieta basal sem suplementação de $\mathrm{P}(0,20 \%$ de $\mathrm{P}$ total $)$ e dieta basal suplementada com fosfato bicálcico para fornecer 15,20 e $25 \mathrm{~g}$ de P/cabeça/dia (Tabelas 1, 2 e 3). As dietas foram fornecidas na forma farelada, e a ingestão de matéria seca $(4,66 \mathrm{~kg} / \mathrm{cabeça} / \mathrm{dia})$ foi previamente estabelecida visando atender às necessidades de ingestão de P. Após vermifugações e pesagens, os animais foram colocados em boxes individuais e submetidos a um período experimental-I, com duração de quinze dias, onde receberam as dietas experimentais para adaptação. No período experimental-II, com duração de sete dias, os animais permaneceram alojados em gaiolas de metabolismo, receberam por injeção via jugular $30 \mathrm{MBq}$ de ${ }^{32} \mathrm{P}\left(\mathrm{Na}_{2} \mathrm{HPO}_{4}\right)$, e foram coletadas amostras de sangue, fezes e urina. Foram determinados os níveis de P inorgânico no plasma e nas fezes (Fiske \& Subbarrow, 1925), como também a atividade radioativa no plasma, fezes e urina, através do efeito Cerenkov (International Atomic Energy Agency, 1979). Foram determinadas as atividades específicas nas fezes e no plasma como porcentagem da atividade injetada, a proporção de $\mathrm{P}$ endógeno, e, conseqüentemente, a disponibilidade biológica ou absorção real (Lofgreen \& Kleiber, 1953). O delineamento experimental foi o de blocos casualizados, com quatro repetições, e foram realizadas a análise de variância e a de regressão polinomial, utilizando o Statistical Analysis System (SAS Institute, 1986). A comparação de médias foi feita pelo teste de Tukey $(\mathrm{P}<0,05)$.

TABELA 1. Composição porcentual da dieta basal.

\begin{tabular}{|c|c|}
\hline Ingredientes & $\%$ na dieta \\
\hline Feno de gramínea moído & 47,0 \\
\hline Milho em grão & 48,0 \\
\hline Farelo de soja & 1,0 \\
\hline Núcleo mineral ${ }^{1}$ & 4,0 \\
\hline Total & 100,0 \\
\hline Matéria seca (\%) & 89,78 \\
\hline Proteína bruta $(\%)$ & 8,15 \\
\hline Cinzas $(\%)$ & 4,65 \\
\hline Fibra bruta (\%) & 14,50 \\
\hline Extrato etéreo $(\%)$ & 3,17 \\
\hline Energia digestível $(\mathrm{kcal} / \mathrm{kg})^{2}$ & 2.500 \\
\hline Fósforo $(\%)$ & 0,22 \\
\hline Cálcio $(\%)$ & 0,20 \\
\hline
\end{tabular}

${ }^{1}$ Sem suplementação de fósforo.

2 Valor estimado de acordo com composição dos alimentos publicados no National Research Council (1989).

TABELA 2. Composição porcentual dos núcleos minerais.

\begin{tabular}{lcccc}
\hline Ingredientes & $\mathrm{N}_{0}$ & $\mathrm{~N}_{15}$ & $\mathrm{~N}_{20}$ & $\mathrm{~N}_{25}$ \\
& $(0 \mathrm{~g} \mathrm{P})$ & $(15 \mathrm{~g}$ de P $)$ & $(20 \mathrm{~g} \mathrm{de} \mathrm{P})$ & $(25 \mathrm{~g} \mathrm{de} \mathrm{P})$ \\
\hline Fosfato bicálcico & - & 15,79 & 28,95 & 42,11 \\
Calcário & 27,63 & 17,66 & 9,35 & 1,04 \\
Sal comum & 17,50 & 17,50 & 17,50 & 17,50 \\
Caulim & 48,10 & 42,28 & 37,43 & 32,58 \\
$\mathrm{MgO}$ & 5,10 & 5,10 & 5,10 & 5,10 \\
Micronutrientes ${ }^{1}$ & 1,67 & 1,67 & 1,67 & 1,67 \\
\hline Total & 100,00 & 100,00 & 100,00 & 100,00 \\
\hline Matéria seca (\%) & 99,70 & 99,35 & 99,09 & 98,70 \\
Cinzas (\%) & 67,44 & 74,82 & 77,68 & 80,82 \\
Fósforo (\%) & 0,08 & 3,12 & $6,01-5,11$ & $9,56-7,43$ \\
Cálcio (\%) & 17,50 & 15,90 & 15,90 & 16,50 \\
\hline
\end{tabular}

${ }^{1}$ Composição dos micronutrientes por kg do produto: $1.500 \mathrm{mg}$ de $\mathrm{Zn}$; $250 \mathrm{mg}$ de $\mathrm{Cu} ; 1.000 \mathrm{mg}$ de Fe; $12,40 \mathrm{mg}$ de Co; $20 \mathrm{mg}$ de I; 2,25 mg de Se; $0,72 \mathrm{~g}$ de $\mathrm{F}$. 


\section{RESULTADOS E DISCUSSÃO}

Os valores médios obtidos quanto aos parâmetros relacionados ao metabolismo de $\mathrm{P}$ estão apresentados na Tabela 4. A concentração plasmática de $\mathrm{P}$ não apresentou diferença $(\mathrm{P}>0,05)$ entre os níveis estudados, e estes foram eficientes em manter valores adequados do elemento no sangue. Houve tendência de os valores de P plasmático aumentarem ligeiramente com os níveis de $\mathrm{P}$ consumido. Da mesma forma, Schryver et al. (1971) e Argenzio et al. (1973) relataram que níveis plasmáticos de $\mathrm{P}$ atingem valores de até $9,8 \mathrm{mg} / \mathrm{kg} / \mathrm{PV}$ quando os animais consumiram altos níveis (em torno de $200 \mathrm{mg} / \mathrm{kg} / \mathrm{PV}$ ) de $\mathrm{P}$, valor este, no entanto, muito superior aos dos utilizados neste trabalho.

TABELA3. Análise bromatológica de rações experimentais com diferentes níveis de $\mathbf{P}$ para eqüinos em crescimento.

\begin{tabular}{|c|c|c|c|c|}
\hline Composição $^{1}$ & $\begin{array}{c}\mathrm{N}_{0} \\
(0 \mathrm{~g} \mathrm{P})\end{array}$ & $\begin{array}{c}\mathrm{N}_{15}{ }^{2} \\
(15 \mathrm{~g} \text { de } \mathrm{P})\end{array}$ & $\begin{array}{c}\mathrm{N}_{20}{ }^{2} \\
(20 \mathrm{~g} \text { de P) }\end{array}$ & $\begin{array}{c}\mathrm{N}_{25}^{2} \\
(25 \mathrm{~g} \text { de } \mathrm{P})\end{array}$ \\
\hline seca $(\%)$ & 89,78 & 89,59 & 89,40 & 88,86 \\
\hline Proteína bruta (\%) & 8,15 & 8,00 & 7,74 & 7,95 \\
\hline Cinzas (\%) & 4,65 & 4,73 & 5,40 & 4,30 \\
\hline Fibra bruta (\%) & 14,50 & 13,85 & 15,43 & 16,19 \\
\hline Extrato etéreo(\%) & 3,17 & 3,01 & 2,99 & 3,05 \\
\hline Fósforo (\%) & 0,22 & 0,33 & 0,41 & 0,50 \\
\hline Cálcio (\%) & 0,88 & 0,83 & 0,58 & 0,63 \\
\hline
\end{tabular}

${ }^{1}$ Em 100\% de matéria seca.

2 O fosfato bicálcico continha $17,66 \%$ de $\mathrm{P}, 23,78 \%$ de Ca e $0,08 \%$ de $\mathrm{F}$.
Considerando o $\mathrm{P}$ excretado na urina, não houve diferença $(\mathrm{P}>0,05)$ entre os tratamentos utilizados no presente trabalho. Os valores obtidos foram baixos, o que indica estarem relacionados com os níveis de P consumido. Segundo Schryver et al. (1971), para baixos níveis $(30 \mathrm{mg} / \mathrm{kg} / \mathrm{PV})$ de consumo de $\mathrm{P}$ a correlação $\mathrm{P}$ consumido/P na urina não é adequada, pois a excreção renal do elemento é baixa ou nula. Por outro lado, Schryver et al. (1971) e Argenzio et al. (1973) relataram que a excreção renal de P aumenta substancialmente (19 a $66 \mathrm{mg} / \mathrm{kg} / \mathrm{PV})$, revestindo-se de grande importância na homeostase do elemento, quando eqüinos receberam altos níveis (100 a $200 \mathrm{mg} / \mathrm{kg} / \mathrm{PV}$ ) de P na dieta.

Os dados de $\mathrm{P}$ total excretado nas fezes mostraram efeito significativo $(\mathrm{P}<0,05)$ entre os tratamentos, e os maiores valores foram com relação a $\mathrm{N}_{20} \mathrm{e}$ $\mathrm{N}_{25}$. Obteve-se uma relação linear entre $\mathrm{P}$ consumido e $\mathrm{P}$ excretado $(\mathrm{r}=0,85 ; \mathrm{P}<0,01)$, expressa pela equação Ptex=3,057+0,673Pcons, ilustrada na Fig. 1. Verificou-se, ainda, que o P total excretado correspondeu, em média, a 73,7\% do $\mathrm{P}$ consumido, o que indica que nos eqüinos a excreção de $\mathrm{P}$ é, na sua maioria, através das fezes, principalmente quando não ocorre alto nível de consumo do elemento.

Quanto aos valores médios de perda endógena fecal, não houve diferença $(\mathrm{P}>0,05)$ entre os tratamentos, apesar de $\mathrm{N}_{15}$ ter apresentado maior valor quanto a este parâmetro. A perda endógena fecal,

TABELA4. Parâmetros associados ao metabolismo de $\mathbf{P}$ de dietas com diferentes níveis do elemento para eqüinos em crescimento ${ }^{1}$.

\begin{tabular}{|c|c|c|c|c|c|}
\hline Parâmetro & $\mathrm{N}_{0}$ & $\mathrm{~N}_{15}$ & $\mathrm{~N}_{20}$ & $\mathrm{~N}_{25}$ & C.V. $(\%)$ \\
\hline $\mathrm{P}$ consumido ${ }^{1}(\mathrm{mg} / \mathrm{kg}$ de $\mathrm{PV})$ & $33,34 \pm 4,46 a$ & $46,82 \pm 6,03 b$ & $59,71 \pm 3,49 \mathrm{c}$ & $77,86 \pm 6,56 \mathrm{~d}$ & 6,86 \\
\hline $\mathrm{P}$ plasma $(\mathrm{mg} \%)$ & $4,42 \pm 0,77$ & $4,50 \pm 0,88$ & $4,60 \pm 0,54$ & $4,71 \pm 0,42$ & 9,44 \\
\hline $\mathrm{P}$ urina $(\mathrm{mg} / \mathrm{kg}$ de $\mathrm{PV})$ & $2,12 \pm 2,20$ & $6,48 \pm 8,18$ & $3,69 \pm 3,80$ & $1,99 \pm 2,15$ & 131,48 \\
\hline P fezes ${ }^{2}$ (mg/kg de PV) & $26,43 \pm 5,58 \mathrm{a}$ & $34,93 \pm 9,29 b$ & $40,48 \pm 13,06 b$ & $57,11 \pm 12,66 b$ & 17,13 \\
\hline $\mathrm{P}$ endógeno (mg/kg de PV) & $9,39 \pm 3,57$ & $11,72 \pm 2,05$ & $10,35 \pm 3,94$ & $9,90 \pm 4,30$ & 23,63 \\
\hline $\mathrm{P}$ absorvido $^{3}(\mathrm{mg} / \mathrm{kg}$ de $\mathrm{PV})$ & $16,29 \pm 2,64 a$ & $22,62 \pm 6,78 \mathrm{ab}$ & $29,43 \pm 13,85 \mathrm{ab}$ & $30,48 \pm 8,84 b$ & 25,59 \\
\hline Disponib. Biológica (\%) & $49,84 \pm 11,36$ & $48,99 \pm 13,58$ & $49,49 \pm 23,03$ & $39,94 \pm 14,11$ & 19,46 \\
\hline $\mathrm{P}$ retido $^{4}(\mathrm{mg} / \mathrm{kg}$ de PV) & $4,78 \pm 4,09$ & $5,42 \pm 4,60$ & $15,54 \pm 10,02$ & $18,76 \pm 9,19$ & 85,8 \\
\hline Meia-vida biológica (horas) & $126,50 \pm 47,74$ & $91,68 \pm 20,57$ & $95,87 \pm 24,19$ & $110,26 \pm 19,55$ & 23,88 \\
\hline Peso vivo (kg) & $319,50 \pm 15,00$ & $320,75 \pm 31,86$ & $324,75 \pm 21,62$ & $325,25 \pm 24,40$ & 3,76 \\
\hline
\end{tabular}

1 Valores seguidos de letras iguais, nas linhas, não diferem entre si pelo teste de Tukey $(\mathrm{P}>0,05)$.

2 Efeito linear dos níveis de $\mathrm{P}(\mathrm{P}<0,05)$ : $\mathrm{y}=3,057+0,673 \mathrm{x}, \mathrm{r}^{2}=0,85$.

${ }^{3}$ Efeito linear dos níveis de $\mathrm{P}(\mathrm{P}<0,05)$ : $\mathrm{y}=5,63+0,34 \mathrm{x}, \mathrm{r}^{2}=0,89$.

${ }^{4}$ Efeito linear dos níveis de $\mathrm{P}(\mathrm{P}<0,05)$ : $\mathrm{y}=-5,287+0,301 \mathrm{x}, \mathrm{r}^{2}=0,44$. 
em média, representou cerca de $28,0 \%$ do $\mathrm{P}$ total excretado nas fezes e não houve relação com o $\mathrm{P}$ consumido da dieta. Isto concorda com Schryver et al. (1971) e Cymbaluk et al. (1989), os quais relataram não haver relação entre $\mathrm{P}$ endógeno fecal e consumo de P em geral.

Com relação à absorção de $\mathrm{P}$, os resultados indicaram efeito de tratamento $(\mathrm{P}<0,05)$ entre os níveis $\mathrm{N}_{0}$ e $\mathrm{N}_{25}$ e uma relação linear entre $\mathrm{P}$ absorvido e P consumido expressa pela equação Pabs $=5,632+0,341$ Pcons $(r=0,89 ; \mathrm{P}<0,01)$, indicada na Fig. 2. A dependência entre nível de ingestão e absorção de P foi também observada em estudos com pôneis, sem afetar, entretanto, a eficiência de absorção real do P (Schryver et al., 1971; Kichura et al.,

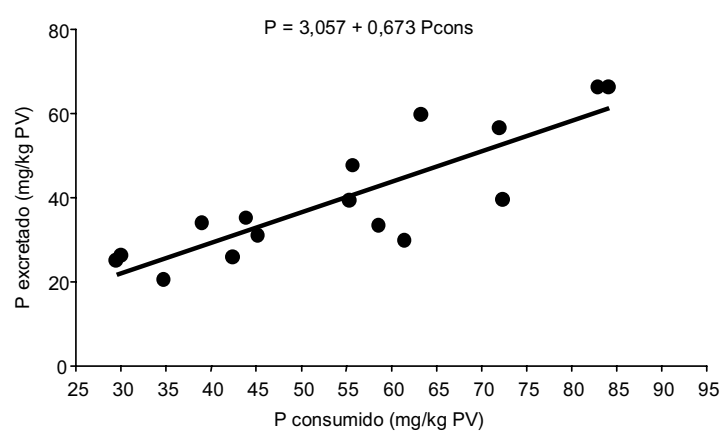

FIG. 1. Fósforo total excretado nas fezes em função do $P$ consumido de dietas com diferentes níveis do elemento para eqüinos em crescimento.



FIG. 2. Fósforo absorvido em função do $P$ consumido de dietas com diferentes níveis do elemento para eqüinos em crescimento.
1983; Cymbaluk \& Christensen, 1986; Cymbaluk et al., 1989).

Não houve efeito $(\mathrm{P}>0,05)$ de tratamentos quanto ao parâmetro disponibilidade biológica (absorção real) neste estudo, e os valores obtidos estão de acordo com as estimativas do National Research Council (1989) e de trabalhos como os de Schryver et al. (1971), Hintz \& Schryver (1972), Hintz et al. (1973) e Cunha (1991), os quais relatam que os fosfatos inorgânicos para eqüinos apresentam absorção real variando de $44 \%$ a $48 \%$.

Considerando-se que no experimento obteve-se uma eficiência média de absorção real de $\mathrm{P}$ da ordem de $47,07 \%$ e perda metabólica fecal média de $10,34 \mathrm{mg} / \mathrm{kg} \mathrm{PV} / \mathrm{dia}$, um potro, nas mesmas condições experimentais, necessitaria de 21,96 mg P/kg PV/dia para manter o balanço da perda metabólica fecal do elemento. Este valor de exigência mínima (manutenção) de P está de acordo com os observados por outros autores, os quais indicam requerimentos mínimos de $\mathrm{P}$ de 21,20 mg/kg PV/dia, ou de 2,1 a 2,5 g P/100 kg PV/dia (Schryver et al., 1971; Robinson \& Slade, 1974; Wolter, 1977; Frape, 1986). Segundo o National Research Council (1989), os requerimentos diários de manutenção situam-se no nível de 22,2 mg P/kg PV/dia.

Nas condições do presente estudo, as exigências de $\mathrm{P}$ em potros com peso vivo médio de $322,56 \mathrm{~kg}$ podem ser calculados considerando-se: deposição de $8 \mathrm{~g}$ de $\mathrm{P} / \mathrm{kg}$ de ganho de peso; 0,45 $\mathrm{kg}$ de ganho de peso e disponibilidade biológica de 47,07\% (8 g x $0,45 \mathrm{~kg} / 0,47 \%)=7,65 \mathrm{~g}$, em adição ao requerimento para manutenção $(322,56 \times 21,96 \mathrm{mg})=7,1 \mathrm{~g}$, totalizando um requerimento diário de $14,75 \mathrm{~g}$ de $\mathrm{P}$. Este valor é ligeiramente inferior ao previsto pelo National Research Council (1989) e Ott (1995), de $16 \mathrm{~g}$ de $\mathrm{P} / \mathrm{animal} / \mathrm{dia}$.

Quanto à retenção de $\mathrm{P}$, obteve-se uma regressão linear entre o $\mathrm{P}$ consumido e o $\mathrm{P}$ retido, expressa pela equação Pret=-5,287+0,301Pcons $(r=0,31 ; P>0,01)$, ilustrada na Fig. 3. A retenção de $\mathrm{P}$ será zero quando o consumo for $17,57 \mathrm{mg} \mathrm{P} / \mathrm{kg} \mathrm{PV}$, valor este que pode também expressar a quantidade mínima de $\mathrm{P}$ que os animais deste trabalho necessitariam para manutenção.

A Fig. 4 permite melhor visualização do comportamento do P no organismo animal. Observou-se que, 




FIG. 3. Retenção de $P$ em função do $P$ consumido de dietas com diferentes níveis do elemento para eqüinos em crescimento.

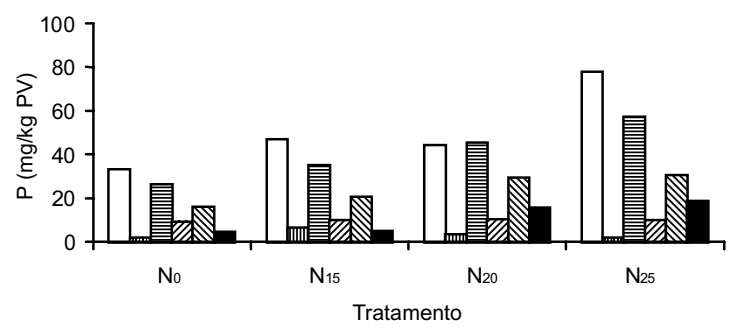

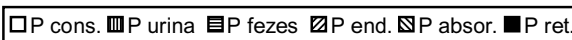

FIG. 4. Distribuição do $P$ em eqüinos em crescimento submetidos a dietas com diferentes níveis do elemento.

aumentando o nível de $\mathrm{P}$ consumido $\left(\mathrm{N}_{25}\right)$, superior às reais exigências do animal, não houve incremento na retenção e absorção do elemento, o que indica que o excesso de $\mathrm{P}$ ingerido foi excretado nas fezes.

\section{CONCLUSÕES}

1. A excreção fecal, a absorção e a retenção de $\mathrm{P}$ dependem do nível de $\mathrm{P}$ consumido.

2. A perda endógena fecal e a disponibilidade biológica de $\mathrm{P}$ não são dependentes do nível de consumo, e apresentam valores médios de $10,34 \mathrm{mg} / \mathrm{kg}$ PV/dia e 47,07\%, respectivamente.

3. Considerando os valores de perda endógena fecal e absorção real de $\mathrm{P}$, os potros necessitam de 21,96 mg P/kg PV/dia para balancear esta perda.
4. Acrescentando-se à exigência de manutenção as exigências para crescimento, os potros necessitam de $45,72 \mathrm{mg} \mathrm{P} / \mathrm{kg} \mathrm{PV} / \mathrm{dia}$ ou $14,75 \mathrm{~g}$ de $\mathrm{P} / \mathrm{animal} / \mathrm{dia}$.

\section{REFERÊNCIAS}

ARGENZIO, R.A.; LOWE, J.E.; HINTZ, H.F.; SCHRYVER, H.F. Calcium and phosphorus homeostasis in horses. Journal of Nutrition, Bethesda, v.104, n.1, p.18-27, 1973.

CUDDEFORD, D. Equine nutritional research: some food for thought? Equine Veterinary Journal, Suffolk, v.23, n.5, p.323-325, 1991.

CUNHA, T.J. Horse feeding and nutrition. 2.ed. San Diego : Academic, 1991. 445p.

CYMBALUK, N.F. Cold housing effects on growth and nutrient demand of young horses. Journal of Animal Science, Champaign, v.68, n.11, p.3152-3162, 1990.

CYMBALUK, N.F.; CHRISTENSEN, D.A. Nutrient utilization of pelleted and unpelleted forages by ponies. Canadian Journal of Animal Science, Ottawa, v.66, n.2, p.237-244, 1986.

CYMBALUK, N.F.; CHRISTISON, G.I.; LEACH, D.H. Nutrient utilization by limit and ad libitum fed growing horses. Journal of Animal Science, Champaign, v.67, n.2, p.414-425, 1989.

FISKE, C.H.; SUBBARROW, Y. The calorimetric determination of phosphorus. Journal of Biological Chemistry, Bethesda, v.66, n.2, p.375-400, 1925.

FRAPE, D.L. Equine nutrition and feeding. New York : Longman Scientific \& Technical, 1986.373p.

HINTZ, H.F.; SCHRYVER, H.F. Availability to ponies of calcium and phosphorus from various supplements. Journal of Animal Science, Champaign, n.34, n.6, p.979-980, 1972.

HINTZ, H.F.; WILLIAMS, A.J.; ROGOFF, J.; SCHRYVER, H.F. Availability of phosphorus in wheat brain when fed to ponies. Journal of Animal Science, Champaign, v.36, n.3, p.522-525, 1973.

INTERNATIONAL ATOMIC ENERGY AGENCY (Vienna, Austria). Laboratory training manual on the use of nuclear techniques in animal research. Vienna, 1979. 300p. 
KICHURA, T.S.; HINTZ, H.F.; SCHRYVER, H.F. Factors influencing endogenous phosphorus losses in ponies. In: EQUINE NUTRITION AND PHYSIOLOGY SYMPOSIUM, 8., 1983, Lexington. Proceedings. Pomona : [s.n.], 1983. p.60-65.

LOFGREEN, G.P.; KLEIBER, M. The availability of the phosphorus in alfalfa hay. Journal of Animal Science, Champaign, v.12, n.2, p.366-371, 1953.

NATIONAL RESEARCH COUNCIL. Board on Agriculture and Natural Resources. Committee on Animal Nutrition (Washington, Estados Unidos). Nutrient requirements of horses. 5.rev.ed. Washington : National Academy of Sciences, 1989. 112p. (Nutrient Requirements of Domestic Animals).
OTT, E.A. Dietary nutrient allowances for horses. Feedstuffs, Minnetonka, v.64, n.29, p.77-80, 1995.

ROBINSON, D.W.; SLADE, L.M. Current status of knowledge on the nutrition of equines. Journal of Animal Science, Champaign, v.39, n.10, p.10451048, 1974.

SAS INSTITUTE (Cary, Estados Unidos). Statistical analysis system: SAS system for linear models. Cary, 1986. 211p.

SCHRYVER, H.F.; HINTZ, H.F.; CRAIG, P.H Phosphorus metabolism in ponies fed varying levels of phosphorus. Journal of Nutrition, Bethesda, v.101, n.19, p.1257-1264, 1971.

WOLTER, R. Alimentación del caballo. 2.ed. Zaragoza : Acribia, 1977. 172p. 